\title{
Stage IV Penile Cancer AJCC v8
}

National Cancer Institute

\section{Source}

National Cancer Institute. Stage IV Penile Cancer A/CC v8. NCI Thesaurus. Code C140087.

Stage IV includes: (T4, Any N, M0); (Any T, N3, M0); (Any T, Any N, M1). T4: Penile cancer with tumor invading into adjacent structures (i.e., scrotum, prostate, pubic bone). N3:

Penile cancer with extranodal extension of lymph node metastases or pelvic lymph node metastases. M1: Distant metastasis present. (AJCC 8th ed.) 